\title{
Timing of Initiation of Renal-Replacement Therapy in Acute Kidney Injury
}

\author{
The STARRT-AKI Investigators, for the Canadian Critical Care Trials Group, \\ the Australian and New Zealand Intensive Care Society Clinical Trials Group, \\ the United Kingdom Critical Care Research Group, the Canadian Nephrology \\ Trials Network, and the Irish Critical Care Trials Group*
}

ABSTRACT

The members of the writing committee (Sean M. Bagshaw, M.D., Ron Wald, M.D., C.M., M.P.H., Neill K.J. Adhikari, M.D., C.M., Rinaldo Bellomo, M.D., Ph.D., Bruno R. da Costa, Ph.D., Didier Dreyfuss, M.D., Bin Du, M.D., Ph.D., Martin P. Gallagher, M.D., Ph.D., Stéphane Gaudry, M.D., Ph.D., Eric A. Hoste, M.D., Ph.D., François Lamontagne, M.D., Michael Joannidis, M.D., Giovanni Landoni, M.D., Kathleen D. Liu, M.D., Ph.D., Daniel F. McAuley, M.D., Shay P. McGuinness, M.D., Javier A. Neyra, M.D., Alistair D. Nichol, M.D., Ph.D., Marlies Ostermann, M.D., Paul M. Palevsky, M.D., Ville Pettilä, M.D., Jean-Pierre Quenot, M.D., Haibo Qiu, M.D., Ph.D., Bram Rochwerg, M.D., Antoine G. Schneider, M.D., Orla M. Smith, R.N., Ph.D., Fernando Thomé, M.D., Ph.D., Kevin E. Thorpe, M.Math., Suvi Vaara, M.D., Ph.D., Matthew Weir, M.D., Amanda Y. Wang, M.D., Ph.D., Paul Young, M.B., Ch.B., Ph.D., and Alexander Zarbock, M.D.) assume responsibility for the overall content and integrity of this article.

The affiliations of the members of the writing committee are listed in the Appendix. Address reprint requests to $\mathrm{Dr}$. Bagshaw at bagshaw@ualberta.ca or to Dr.Wald at ron.wald@unityhealth.to.

*A list of the group members in the STARRT-AKI trial is provided in the Supplementary Appendix, available at NEJM.org.

Drs. Bagshaw and Wald contributed equally to this article.

This article was updated on July 16,2020 , at NEJM.org.

N EnglJ Med 2020;383:240-51.

DOI: 10.1056/NEJMoa2000741

Copyright (c) 2020 Massachusetts Medical Society.

\section{BACKGROUND}

Acute kidney injury is common in critically ill patients, many of whom receive renal-replacement therapy. However, the most effective timing for the initiation of such therapy remains uncertain.

\section{METHODS}

We conducted a multinational, randomized, controlled trial involving critically ill patients with severe acute kidney injury. Patients were randomly assigned to receive an accelerated strategy of renal-replacement therapy (in which therapy was initiated within 12 hours after the patient had met eligibility criteria) or a standard strategy (in which renal-replacement therapy was discouraged unless conventional indications developed or acute kidney injury persisted for $>72$ hours). The primary outcome was death from any cause at 90 days.

\section{RESULTS}

Of the 3019 patients who had undergone randomization, 2927 (97.0\%) were included in the modified intention-to-treat analysis (1465 in the accelerated-strategy group and 1462 in the standard-strategy group). Of these patients, renal-replacement therapy was performed in 1418 (96.8\%) in the accelerated-strategy group and in $903(61.8 \%)$ in the standard-strategy group. At 90 days, death had occurred in 643 patients $(43.9 \%)$ in the accelerated-strategy group and in $639(43.7 \%)$ in the standard-strategy group (relative risk, 1.00; 95\% confidence interval [CI], 0.93 to 1.09; $\mathrm{P}=0.92$ ). Among survivors at 90 days, continued dependence on renalreplacement therapy was confirmed in 85 of 814 patients (10.4\%) in the acceleratedstrategy group and in 49 of 815 patients $(6.0 \%)$ in the standard-strategy group (relative risk, 1.74; 95\% CI, 1.24 to 2.43). Adverse events occurred in 346 of 1503 patients (23.0\%) in the accelerated-strategy group and in 245 of 1489 patients $(16.5 \%)$ in the standard-strategy group $(\mathrm{P}<0.001)$.

\section{CONCLUSIONS}

Among critically ill patients with acute kidney injury, an accelerated renal-replacement strategy was not associated with a lower risk of death at 90 days than a standard strategy. (Funded by the Canadian Institutes of Health Research and others; STARRT-AKI ClinicalTrials.gov number, NCT02568722.) 
CUTE KIDNEY INJURY IS A COMMON complication in patients admitted to an intensive care unit (ICU) and is associated with a high risk of death or major complications and a high level of resource use. ${ }^{1}$ Many patients who are seriously ill undergo renal-replacement therapy; however, the appropriate timing for the initiation of such therapy remains uncertain. When acute kidney injury is complicated by major metabolic disorders (e.g., acidosis, hyperkalemia, and uremia) and fluid disturbances that can be treated with renal-replacement therapy, there is general consensus that such therapy should be initiated. ${ }^{2,3}$ However, when severe acute kidney injury is not accompanied by one of these complications, the benefits of renal-replacement therapy are unclear. ${ }^{4-9}$

The initiation of renal-replacement therapy before the onset of major complications has conceivable advantages for patients with severe acute kidney injury. Such therapy can restore and maintain acid-base homeostasis, mitigate fluid accumulation, and reduce exposure to the metabolic hazards of untreated acute kidney injury. ${ }^{10}$ However, wide adoption of early renal-replacement therapy in these patients may lead to initiation in those who would have survived and recovered kidney function without such therapy. ${ }^{5}$ Furthermore, given the risks of renal-replacement therapy, establishing whether earlier initiation can improve outcomes is important for patient care. Randomized trials that have compared early and delayed strategies for starting renal-replacement therapy in critically ill patients with acute kidney injury have shown discrepant findings. ${ }^{4-7}$

Here, we report the results of a multinational, randomized, open-label, controlled trial (STARRTAKI [Standard versus Accelerated Initiation of Renal-Replacement Therapy in Acute Kidney Injury]) to compare the effect of two strategies (accelerated initiation vs. standard initiation) in critically ill patients with acute kidney injury. We hypothesized that an accelerated strategy for renal-replacement therapy would result in a lower risk of death from any cause at 90 days than a standard strategy.

METHODS

TRIAL DESIGN AND OVERSIGHT

We conducted STARRT-AKI at 168 hospitals in 15 countries. A complete list of participating sites is provided in the Supplementary Appendix, available with the full text of this article at NEJM.org. The protocol along with its statistical analysis plan (also available at NEJM.org) was published previously. ${ }^{11,12}$ The investigators designed the trial, wrote the manuscript, and vouch for the accuracy and completeness of the data and the analyses, for the adherence of the trial to the protocol, and for the complete reporting of adverse events. The funding organizations and partners were not involved in the design, implementation, or management of the trial, in the analysis of the data, or in the decision to submit the manuscript for publication.

Approval for the conduct of the trial was obtained from the institutional review board at each participating site. We obtained written informed consent from patients or substitute decision makers unless the requirement for informed consent was waived, according to local standards and legislation. An independent data and safety monitoring board provided trial oversight. Interim analyses to evaluate the primary outcome were performed when enrollment reached 25\%, 50\%, and $75 \%$ of the target (Table S1 in the Supplementary Appendix).

\section{SELECTION OF PATIENTS}

Patients were eligible if they were 18 years or older and had been admitted to an ICU with kidney dysfunction (serum creatinine level, $\geq 1.13 \mathrm{mg}$ per deciliter [100 $\mu$ mol per liter] in women and $\geq 1.47 \mathrm{mg}$ per deciliter [130 $\mu \mathrm{mol}$ per liter] in men) and severe acute kidney injury that was categorized as stage 2 or 3 of the Kidney Disease: Improving Global Outcomes (KDIGO) classification (in which stages range from 1 to 3 , with higher stages indicating greater severity). This determination of kidney injury was defined by a doubling of the serum creatinine level from baseline, a serum creatinine level of $4 \mathrm{mg}$ per deciliter (354 $\mu \mathrm{mol}$ per liter) or more with an increase of $0.3 \mathrm{mg}$ per deciliter (27 $\mu \mathrm{mol}$ per liter) from baseline, or a urine output of less than $6 \mathrm{ml}$ per kilogram of body weight during the preceding 12 hours. The exclusion criteria were focused on emergency indications, previous renal-replacement therapy, advanced chronic kidney disease, and uncommon causes of acute kidney injury (as detailed in Table S2). The presence of all the inclusion criteria and none of the first eight exclusion criteria established provisional eligibility (Fig. S1). 
To confirm full eligibility, patients' attending physicians were asked to affirm clinical equipoise by noting the absence of any circumstances that would mandate either immediate initiation of renal-replacement therapy or a deferral of such therapy because of clinical judgment regarding the likelihood of imminent recovery of kidney function. After a determination of full eligibility, a 12-hour window was allotted for consent (as applicable), randomization, and the initiation of renal-replacement therapy for patients in the accelerated-therapy group.

At baseline, we evaluated patients using the Simplified Acute Physiology Score (SAPS) II on a scale that ranges from 0 to 163 , with higher scores indicating more severe disease and a higher risk of death. Patients were also evaluated with the Sequential Organ Failure Assessment (SOFA) on a scale that ranges from 0 to 24 , with higher scores indicating more severe disease and a higher risk of death.

\section{RANDOMIZATION AND INTERVENTIONS}

Patients were randomly assigned in a 1:1 ratio to receive a strategy of accelerated or standard initiation of renal-replacement therapy. Randomization with variable block size ( 2 and 4 ) and site stratification were implemented with the use of a centralized Web-based platform.

In the accelerated-strategy group, clinicians were to start renal-replacement therapy as soon as possible and within 12 hours after patients had met full eligibility criteria. In the standardstrategy group, clinicians were discouraged from initiating renal-replacement therapy until the development of one or more of the following criteria: a serum potassium level of $6.0 \mathrm{mmol}$ or more per liter, a pH of 7.20 or less or a serum bicarbonate level of $12 \mathrm{mmol}$ per liter or less, evidence of severe respiratory failure based on a ratio of the partial pressure of arterial oxygen to the fraction of inspired oxygen of 200 or less and clinical perception of volume overload, or persistent acute kidney injury for at least 72 hours after randomization. For patients in the standardstrategy group, clinicians were not obligated to initiate renal-replacement therapy. Similarly, clinicians had discretion to initiate such therapy at any time if they perceived that deferral was no longer in the patient's best interest.

The protocol provided recommendations for the delivery of renal-replacement therapy that aligned with recognized international guidelines. ${ }^{13}$ Discontinuation of renal-replacement therapy occurred at the time of recovery of kidney function, withdrawal of life-sustaining support, or death.

\section{OUTCOMES}

The primary outcome was death from any cause at 90 days after randomization. Key secondary outcomes at 90 days were dependence on renalreplacement therapy; a composite of death or dependence on renal-replacement therapy; and a major adverse kidney event, which was defined as death, dependence on renal-replacement therapy, or a sustained reduction in kidney function (i.e., an estimated glomerular filtration rate [eGFR] of $<75 \%$ of the baseline value ${ }^{14}$ ). Additional prespecified secondary outcomes included death in the ICU at 28 days or during hospitalization; the number of days free of renal-replacement therapy at 90 days; the number of ventilatorfree and vasoactive-free days at 28 days $^{4,15}$; the length of hospitalization and hospitalization-free days at 90 days; and health-related quality-oflife, as assessed at 90 days by means of the European Quality of Life-5-Dimensions 5-Level questionnaire (EQ-5D-5L; scores range from 0 to 100 , with higher scores indicating a better quality of life). ${ }^{16}$

Adverse events related to renal-replacement therapy and vascular access were reported through 14 days among all the patients who had undergone randomization and remained in the ICU. All serious adverse events were reviewed by the cochairs of the trial and by the chair of the data and safety monitoring board within 48 hours after notification.

\section{STATISTICAL ANALYSIS}

We performed all analyses in accordance with the published statistical analysis plan using a modified intention-to-treat principle after the exclusion of patients who had withdrawn consent, had been lost to follow-up, or had undergone randomization but had subsequently been found to be ineligible. ${ }^{11}$ On the basis of an assumed 90 -day mortality of $40 \%$ in the standardstrategy group, we calculated that the enrollment of 2866 patients would provide a power of $90 \%$ to detect a $15 \%$ relative between-group difference (absolute difference, 6 percentage points) in the primary outcome at a two-sided signifi- 
cance level of 0.05 , while allowing for $3 \%$ attrition (withdrawal of consent, loss to follow-up, or crossover). ${ }^{6,17}$ To compensate for such attrition, we revised recruitment to 3000 patients. In the United Kingdom, enrollment was extended to meet local recruitment targets. The expansion in recruitment was approved by the data and safety monitoring board.

We evaluated the primary outcome of death at 90 days using a chi-square test, with the results reported as a relative risk and absolute risk difference with $95 \%$ confidence intervals. Death at 90 days was also reported in an adjusted logistic-regression analysis as an odds ratio with $95 \%$ confidence intervals and in a Kaplan-Meier timeto-event analysis in which data were censored at 90 days and compared with the use of a log-rank test. We performed prespecified exploratory analyses of the primary outcome (Table S3) and conducted prespecified subgroup analyses of the primary outcome for six variables: sex, baseline eGFR, baseline SAPS II, surgical admission, the presence of sepsis, and geographic region. ${ }^{18}$

We reported secondary outcomes - including dependence on renal-replacement therapy, a composite of death or dependence on renalreplacement therapy, and a major adverse kidney event at 90 days - as unadjusted relative risks with $95 \%$ confidence intervals and performed between-group comparisons using a chi-square test. We used inverse probability weighting and multinomial regression to account for postrandomization selection bias when evaluating continuing dependence on renal-replacement therapy (Table S3). We used a t-test to compare serum creatinine levels and eGFR values among survivors at 90 days. We calculated medians and interquartile ranges to summarize the number of days that patients were alive and free of renalreplacement therapy, the use of vasoactive drugs or mechanical ventilation, ICU stay, hospital stay, and quality of life according to treatment group. We used linear regression to perform betweengroup comparisons and reported the results as mean differences and $95 \%$ confidence intervals. The statistical analysis plan did not include a provision that secondary analyses would be corrected for multiple comparisons. ${ }^{11}$ As such, the $95 \%$ confidence intervals have not been adjusted for multiplicity and should not be used to infer definitive treatment effects for secondary outcomes. All statistical analyses were performed with the use of Stata software, version 15, and $\mathrm{R}$ software, version 3.6.2. ${ }^{19}$

\section{RESULTS}

\section{PATIENTS}

From October 2015 through September 2019, a total of 11,852 patients met provisional eligibility. Of these patients, 3019 were randomly assigned to receive either an accelerated strategy for the initiation of renal-replacement therapy (1512 patients) or a standard strategy (1507 patients) (Fig. S2). The number of patients who were subsequently determined to be ineligible to participate were $31(2.1 \%)$ in the accelerated-strategy group and 19 (1.3\%) in the standard-strategy group (Table $\mathrm{S} 4)$. In the accelerated-strategy group, 9 patients $(0.6 \%)$ withdrew consent and $7(0.5 \%)$ were lost to follow-up; the corresponding numbers in the standard-strategy group were 18 (1.2\%) and $8(0.5 \%)$. Thus, 2927 patients (1465 in the accelerated-strategy group and 1462 in the standard-strategy group) were included in the modified intention-to-treat analysis.

Baseline characteristics were well balanced in the two groups (Table 1 and Table S5). In the entire population, chronic kidney disease was present in 1284 patients (43.9\%); 965 patients (33.0\%) had been admitted to undergo surgery, 1689 patients (57.7\%) had sepsis, and the mean $( \pm$ SD) SAPS II and SOFA scores were $58.8 \pm 17.4$ and 11.7 \pm 3.6 , respectively.

\section{INITIATION OF RENAL-REPLACEMENT THERAPY}

In the accelerated-strategy group, renal-replacement therapy was initiated at a median of 6.1 hours (interquartile range, 3.9 to 8.8 ) after the determination of full eligibility in 1418 of 1465 patients $(96.8 \%)$. In the standard-strategy group, such therapy was initiated in 903 of 1462 patients $(61.8 \%)$ at a median of 31.1 hours (interquartile range, 19.0 to 71.8 ) after the determination of full eligibility (Fig. S3). The characteristics of the patients at the time of renal-replacement therapy and features of the initial prescription are provided in Tables S6 and S7. Among the patients in the standard-strategy group who underwent renal-replacement therapy, 597 of 903 (66.1\%) fulfilled at least one prespecified indication for the initiation of such therapy. At the time of initiation, the SOFA score, serum creatinine level, blood urea nitrogen level, and positive 


\begin{tabular}{|c|c|c|}
\hline Characteristic & $\begin{array}{l}\text { Accelerated Strategy } \\
\qquad(\mathrm{N}=1465)\end{array}$ & $\begin{array}{l}\text { Standard Strategy } \\
\quad(N=1462)\end{array}$ \\
\hline Age $-y r$ & $64.6 \pm 14.3$ & $64.7 \pm 13.4$ \\
\hline Female sex - no. (\%) & $470(32.1)$ & 467 (31.9) \\
\hline Weight — kg & $88.0 \pm 27.4$ & $88.0 \pm 25.1$ \\
\hline Serum creatinine $-\mathrm{mg} / \mathrm{dl}^{\dagger}$ & $1.4 \pm 1.0$ & $1.3 \pm 1.0$ \\
\hline Estimated glomerular filtration rate $-\mathrm{ml} / \mathrm{min} / 1.73 \mathrm{~m}^{2}+$ & $66.0 \pm 29.8$ & $67.3 \pm 29.8$ \\
\hline \multicolumn{3}{|l|}{ Preexisting conditions — no./total no. (\%) } \\
\hline Chronic kidney disease & $658 / 1465(44.9)$ & $626 / 1462(42.8)$ \\
\hline Hypertension & $814 / 1465(55.6)$ & $823 / 1462(56.3)$ \\
\hline Diabetes mellitus & $439 / 1465(30.0)$ & $459 / 1461(31.4)$ \\
\hline Heart failure & $204 / 1465$ (13.9) & 204/1461 (14.0) \\
\hline Coronary artery disease & $320 / 1465(21.8)$ & $328 / 1461(22.5) \sqrt{ }$ \\
\hline Liver disease & $172 / 1465(11.7)$ & 165/1461 (11.3) \\
\hline Metastatic cancer & $77 / 1465(5.3)$ & $84 / 1462(5.7)$ \\
\hline Hematologic cancer & $87 / 1465$ (5.9) & $83 / 1462(5.7)$ \\
\hline HIV infection or AIDS & $13 / 1465$ (0.9) & $13 / 1462(0.9)$ \\
\hline \multicolumn{3}{|l|}{ Admission category — no. (\%) } \\
\hline Scheduled surgery & $207(14.1)$ & $184(12.6)$ \\
\hline Unscheduled surgery & $285(19.5)$ & $289(19.8)$ \\
\hline Medical & $973(66.4)$ & 989 (67.6) \\
\hline \multicolumn{3}{|l|}{$\begin{array}{l}\text { Hospital-acquired risk factor for AKI in previous wk } \\
\quad-\text { no./total no. (\%) }\end{array}$} \\
\hline Cardiopulmonary bypass & $112 / 1465(7.6)$ & $118 / 1462(8.1)$ \\
\hline Aortic aneurysm repair & $71 / 1465$ (4.8) & $74 / 1461(5.1)$ \\
\hline Other vascular surgery & $76 / 1465(5.2)$ & $77 / 1462(5.3)$ \\
\hline Major trauma & $62 / 1465(4.2)$ & $55 / 1462(3.8)$ \\
\hline Obstetric complication & $5 / 1465(0.3)$ & $5 / 1462(0.3)$ \\
\hline Exposure to radiocontrast material & $382 / 1463(26.1)$ & $375 / 1460(25.7)$ \\
\hline Receipt of aminoglycoside & $154 / 1463(10.5)$ & $148 / 1458(10.2)$ \\
\hline Receipt of amphotericin B & $9 / 1464(0.6)$ & $12 / 1460(0.8)$ \\
\hline \multicolumn{3}{|l|}{ Clinical condition at randomization } \\
\hline Sepsis - no. (\%) & $855(58.4)$ & $834(57.0)$ \\
\hline Septic shock - no. (\%) & $640(43.7)$ & $643(44.0)$ \\
\hline SAPS II value & $58.1 \pm 17.4$ & $59.4 \pm 17.4$ \\
\hline SOFA score $\|$ & $11.6 \pm 3.6$ & $11.8 \pm 3.6$ \\
\hline Mechanical ventilation — no. (\%) & $1103(75.3)$ & $1148(78.5)$ \\
\hline Vasoactive support — no. (\%) & $1008(68.8)$ & $1052(72.0)$ \\
\hline Serum creatinine $-\mathrm{mg} / \mathrm{dl}$ & $3.6 \pm 1.7$ & $3.4 \pm 1.6$ \\
\hline Serum potassium $-\mathrm{mmol} / \mathrm{liter}$ & $4.5 \pm 0.8$ & $4.5 \pm 0.8$ \\
\hline Serum bicarbonate - mmol/liter & $19.7 \pm 4.7$ & $19.5 \pm 4.5$ \\
\hline Median urinary output (IQR) $-\mathrm{ml} / 24 \mathrm{hr}$ & $450(190-945)$ & $478(187-975)$ \\
\hline Oliguria or anuria — no./total no. (\%) †† & $647 / 1415(45.7)$ & $618 / 1420(43.5)$ \\
\hline
\end{tabular}




\begin{tabular}{|lcc|}
\hline Table 1. (Continued.) & \\
\hline \\
Characteristic & $\begin{array}{c}\text { Accelerated Strategy } \\
(\mathbf{N}=1465)\end{array}$ & $\begin{array}{c}\text { Standard Strategy } \\
\text { (N=1462) }\end{array}$ \\
Median cumulative fluid balance (IQR) $-\mathrm{mltr}$ & $2581(820-5362)$ & $2819(836-5603)$
\end{tabular}

* Plus-minus values are means \pm SD. Listed are data in the modified intention-to-treat population. To convert the values for creatinine to micromoles per liter, multiply by 88.4. AIDS denotes acquired immunodeficiency syndrome, AKI acute kidney injury, HIV human immunodeficiency virus, and IQR interquartile range.

$\dagger$ At baseline, the serum creatinine level was defined as the most recent outpatient level obtained during the year preceding the current hospitalization. If this value was not available, the lowest serum creatinine level obtained during the current hospitalization was used to establish the baseline.

+ The estimated glomerular filtration rate was calculated with the use of the Chronic Kidney Disease Epidemiology Collaboration equation, which incorporates the baseline serum creatinine level, age, sex, and black race.

$\int$ Data in this category were missing for 1 patient in the standard-strategy group.

I Results for the Simplified Acute Physiology Score (SAPS) II range from 0 to 163, with higher scores indicating more severe disease and a higher risk of death.

|| Scores on the Sequential Organ Failure Assessment (SOFA) range from 0 to 24, with higher scores indicating more severe disease and a higher risk of death.

$* *$ Data regarding urinary output were available for 2835 of 2927 patients (96.9\%) who had collection of data for at least 24 hours.

t广 Oliguria was defined as a urinary output of less than $400 \mathrm{ml}$ per 24-hour period.

Data regarding cumulative fluid balance after admission to an intensive care unit were available for 2738 of $2927 \mathrm{pa}$ tients (93.5\%): 1378 in the accelerated-strategy group and 1360 in the standard-strategy group..

fluid balance were higher among the patients in the standard-strategy group than among those in the accelerated-strategy group.

\section{PRIMARY OUTCOME}

In the modified intention-to-treat analysis, death at 90 days occurred in 643 patients $(43.9 \%)$ in the accelerated-strategy group and in 639 (43.7\%) in the standard-strategy group (relative risk, 1.00; $95 \%$ confidence interval [CI], 0.93 to 1.09 ), for an absolute risk difference of 0.2 percentage points ( $95 \% \mathrm{CI},-0.3$ to $0.4 ; \mathrm{P}=0.92$ ) (Table 2, Fig. 1, and Fig. S4). Results were similar in the adjusted analysis (adjusted odds ratio, 1.05; $95 \% \mathrm{CI}, 0.90$ to 1.23 ).

\section{SECONDARY OUTCOMES}

Among the patients who were alive at 90 days, continued dependence on renal-replacement therapy was determined in 85 of 814 (10.4\%) in the accelerated-strategy group and in 49 of 815 $(6.0 \%)$ in the standard-strategy group (relative risk, $1.74 ; 95 \%$ CI, 1.24 to 2.43 ). This result was robust after inverse probability weighting and in a multinomial analysis (Table S8).

There was no meaningful between-group difference in the composite of death or dependence on renal-replacement therapy, major adverse kidney events at 90 days, death in the ICU at 28 days, or length of hospitalization (Table 2). In addi- tion, there were no substantial between-group differences in the causes of death (Table S9). In each group, patients had a similar number of days free of renal-replacement therapy at 90 days; there were marginally fewer days of renal-replacement therapy among the patients in the accelerated-strategy group than among those in the standard-strategy group $(-0.48$ days; $95 \% \mathrm{CI}$, -0.82 to -0.14$)$. Patients in the acceleratedstrategy group had a shorter ICU stay than those in the standard-strategy group, but there was no evidence of large between-group differences in the number of ventilator-free days, vasoactive-free days, or ICU-free days at 28 days. Patients in the accelerated-strategy group had a higher risk of rehospitalization (relative risk, 1.23; $95 \% \mathrm{CI}$, 1.02 to 1.49), but there was no evidence of a large between-group difference in the number of hospitalization-free days at 90 days. Among survivors, measures of quality of life and functional domains were similar.

\section{SUBGROUP ANALYSES}

There was no evidence of substantial betweengroup heterogeneity of treatment effect regarding 90-day mortality across prespecified subgroups (Fig. 2 and Tables S10 and S11). Similarly, there was no evidence of substantial heterogeneity of treatment effect on 90-day mortality across SAPS II scores at the time of enrollment (Fig. S5). 
Table 2. Primary and Secondary Outcomes.*

\begin{tabular}{|c|c|c|c|}
\hline & $\begin{array}{l}\text { Accelerated } \\
\text { Strategy } \\
(\mathrm{N}=1465)\end{array}$ & $\begin{array}{c}\text { Standard } \\
\text { Strategy } \\
(\mathrm{N}=1462)\end{array}$ & $\begin{array}{c}\text { Relative Risk } \\
\text { or Difference } \\
\quad(95 \% \mathrm{Cl})\end{array}$ \\
\hline \multicolumn{4}{|l|}{ Primary outcome } \\
\hline Death from any cause at 90 days - no. $(\%) \dagger$ & $643(43.9)$ & $639(43.7)$ & $1.00(0.93$ to 1.09$) \div$ \\
\hline \multicolumn{4}{|l|}{ Secondary outcomes } \\
\hline RRT dependence among survivors at 90 days - no./total no. (\%) & $85 / 814(10.4)$ & $49 / 815(6.0)$ & $1.74(1.24$ to 2.43$) \div$ \\
\hline Death or RRT dependence at 90 days — no./total no. (\%) & $728 / 1457(50.0)$ & $688 / 1454(47.3)$ & $1.06(0.98$ to 1.14$) \div$ \\
\hline Major adverse kidney events at 90 days — no./total no. (\%) & $867 / 1131(76.7)$ & $860 / 1115(77.1)$ & $0.99(0.95$ to 1.04$) \div$ \\
\hline Serum creatinine at 90 days $-\mathrm{mg} / \mathrm{dl} \sqrt{ }$ & $1.20 \pm 1.00$ & $1.23 \pm 1.00$ & $-0.03(-0.11$ to 0.06$) 9$ \\
\hline \multicolumn{4}{|l|}{ Estimated glomerular filtration rate\| } \\
\hline At 90 days $-\mathrm{ml} / \mathrm{min} / 1.73 \mathrm{~m}^{2}$ & $65 \pm 30$ & $64 \pm 31$ & $0.31(-3.88$ to 4.49$) 9$ \\
\hline $\begin{array}{l}\text { Reduction of }>25 \% \text { from baseline at } 90 \text { days - no./ } \\
\text { total no. (\%) }\end{array}$ & $139 / 403(34.5)$ & $172 / 427(40.3)$ & $0.86(0.72$ to 1.02$) \div$ \\
\hline \multicolumn{4}{|l|}{ Death from any cause - no./total no. (\%) } \\
\hline At any time in the ICU & $461 / 1464(31.5)$ & $468 / 1462(32.0)$ & 0.98 (0.88 to 1.09$) \div$ \\
\hline At 28 days & $538 / 1465(36.7)$ & $523 / 1462(35.8)$ & 1.03 (0.93 to 1.13$) \div$ \\
\hline During hospitalization & $552 / 1458(37.9)$ & $546 / 1459(37.4)$ & $1.01(0.92$ to 1.11$) \div$ \\
\hline \multicolumn{4}{|l|}{ Use of health services } \\
\hline \multicolumn{4}{|l|}{ Median no. of days of use (IQR) } \\
\hline RRT-free days at 90 days $* *$ & 50 (0 to 87$)$ & 64 (0 to 90$)$ & $-2.62(-5.66$ to 0.42$) 9$ \\
\hline RRTit & $4(2$ to 8$)$ & 5 (3 to 9$)$ & $-0.48(-0.82$ to -0.14$) 9$ \\
\hline Continuous RRTit & $4(3$ to 8$)$ & $5(3$ to 8$)$ & $-0.40(-0.78$ to -0.02$) 9$ \\
\hline Sustained low-efficiency dialysis $\rangle^{\dagger} \dagger$ & $2(1$ to 4$)$ & $2(1$ to 4$)$ & $0.15(-0.65$ to 0.96$) 9$ \\
\hline Intermittent hemodialysis $† \dagger$ & 2 (1 to 4$)$ & $3(2$ to 5$)$ & $-0.45(-0.80$ to -0.09$) 9$ \\
\hline \multicolumn{4}{|l|}{ Median length of stay in ICU (IQR) - days } \\
\hline Survivors & $9(5$ to 16$)$ & $10(5$ to 19$)$ & $-1.58(-2.90$ to -0.26$) 9$ \\
\hline Nonsurvivors & 7 (3 to 13$)$ & 7 (4 to 15$)$ & $-1.33(-2.56$ to -0.09$) 9$ \\
\hline \multicolumn{4}{|l|}{ Median length of hospital stay (IQR) - days } \\
\hline Survivors & 28 (16 to 50$)$ & 29 (17 to 54$)$ & $-1.23(-3.87$ to 1.41$) 9$ \\
\hline Nonsurvivors & $8(3$ to 18$)$ & $9(4$ to 19$)$ & $-0.99(-2.66$ to 0.67$) 9$ \\
\hline Median no. of ventilator-free days at 28 days (IQR) & $13(0$ to 24$)$ & $12(0$ to 24$)$ & $0.50(-0.34$ to 1.35$) 9$ \\
\hline Median no. of days free of vasoactive agents at 28 days (IQR) & 21 (0 to 26$)$ & $20(0$ to 26$)$ & $0.31(-0.57$ to 1.18$) 9$ \\
\hline Median no. of days out of ICU at 28 days (IQR) & $8(0$ to 21$)$ & $4(0$ to 20$)$ & $0.69(-0.06$ to 1.43$) 9$ \\
\hline Median no. of days out of hospital at 90 days (IQR) & $10(0$ to 65$)$ & $9(0$ to 64$)$ & $0.55(-1.82$ to 2.91$) 9$ \\
\hline Rehospitalization at 90 days - no./total no. (\%) & $191 / 913(20.9)$ & $156 / 916(17.0)$ & $1.23(1.02$ to 1.49$) \div$ \\
\hline \multicolumn{4}{|l|}{ Health-related quality of life } \\
\hline \multicolumn{4}{|l|}{ Median score on EQ-5D-5L at 90 days (IQR) } \\
\hline \multicolumn{4}{|l|}{ Descriptive system } \\
\hline Mobility & 2 (1 to 3$)$ & $2(1$ to 3$)$ & $-0.07(-0.23$ to 0.08$) 9$ \\
\hline Self care & $1(1$ to 3$)$ & $1(1$ to 3$)$ & $-0.10(-0.25$ to 0.05$) 9$ \\
\hline Usual activities & 2 (1 to 3$)$ & 2 (1 to 4$)$ & $-0.15(-0.31$ to 0.01$)$ \\
\hline Pain or discomfort & 2 (1 to 3$)$ & 2 (1 to 3$)$ & $-0.04(-0.17$ to 0.08$)$ \\
\hline Anxiety or depression & 1 (1 to 3$)$ & 2 (1 to 3$)$ & $-0.06(-0.19$ to 0.07$)$ \\
\hline
\end{tabular}


Table 2. (Continued.)

EQ-VASIS

Score of $>4$ on Clinical Frailty Scale at 90 days - no. (\%) 99

\section{Accelerated \\ Strategy \\ $(\mathrm{N}=1465)$}

70 (50 to 80$)$

$213 / 655(32.5)$

$\begin{gathered}\text { Standard } \\ \text { Strategy } \\ (\mathbf{N}=1462)\end{gathered}$
$70(50$ to 80$)$
$227 / 647(35.1)$

Relative Risk

or Difference

$(95 \% \mathrm{CI})$

$0.11(-2.55$ to 2.76$) 9$

$0.93(0.80$ to 1.08$) \div$

* Plus-minus values are means \pm SD. ICU denotes intensive care unit, and RRT renal-replacement therapy.

$\dagger \mathrm{P}=0.92$ for the primary outcome. P values were not calculated for the secondary outcomes because comparisons were not adjusted for multiple comparisons.

$\mp$ This value is expressed as a relative risk.

$\int$ Data regarding serum creatinine levels are for survivors at 90 days who were not dependent on kidney-replacement therapy. Of these 1511 patients, data were available for 830 (54.9\%): 403 patients in the accelerated-strategy group and 427 patients in the standard-strategy group.

II This value is expressed as a mean difference.

$\|$ The estimated glomerular filtration rate was calculated with the Chronic Kidney Disease Epidemiology Collaboration equation.

w: Data regarding RRT-free days are provided for 2900 of 2927 (99.0\%) patients: 1448 in the accelerated-strategy group and 1452 in the standard-strategy group.

$T$ Included in this category are patients who received renal-replacement therapy.

+ On the European Quality of Life-5-Dimensions 5-Level questionnaire (EQ-5D-5L), scores for each of the five domains of the descriptive system range from 1 to 5 , with higher scores representing greater impairment or worse function. Scores at 90 days were available for 1164 of 1645 patients $(70.8 \%)$ for the mobility domain (581 patients in the accelerated-strategy group and 583 patients in the standard-strategy group); 1165 of 1645 (70.8\%) for the self-care domain (582 patients and 583 patients, respectively); 1163 of 1645 (70.7\%) for the usual activities domain (581 patients and 582 patients, respectively); 1162 of 1645 (70.6\%) for the pain or discomfort domain (580 patients and 582 patients, respectively); and 1153 of 1645 (70.1\%) for the anxiety or depression domain (576 patients and 577 patients, respectively).

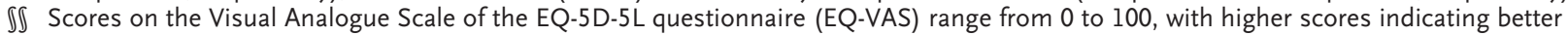
health. EQ-VAS scores were available for 1103 of 1645 survivors (67.0\%) at 90 days: 555 patients in the accelerated-strategy group and 548 in the standard-strategy group.

91 Scores on the Clinical Frailty Scale range from 1 to 9, with higher scores indicating a higher degree of frailty. A score of more than 4 is the threshold for the definition of frailty. Scores on this scale at 90 days were available for 1302 of 1645 survivors (79.1\%).

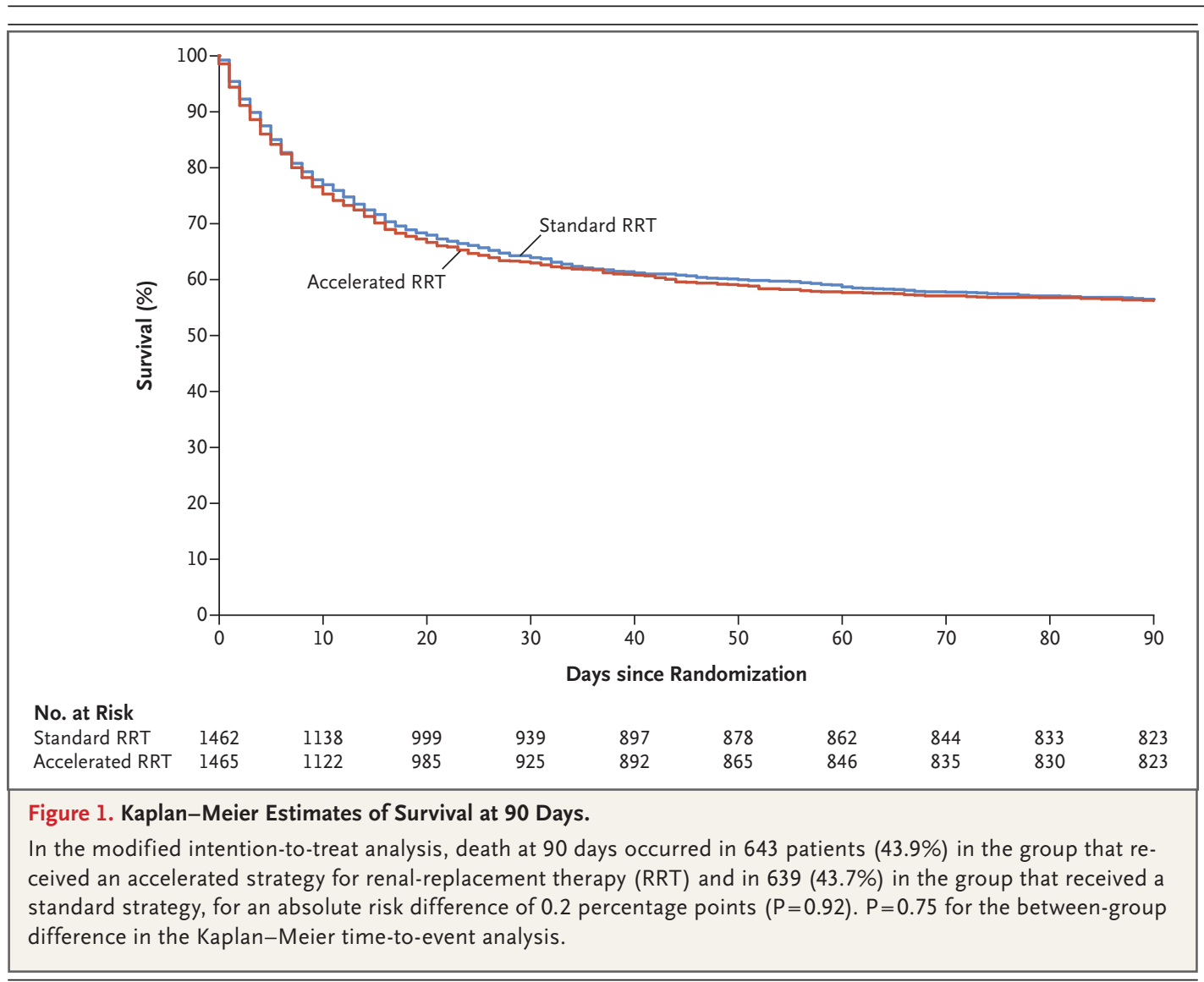

N ENGLJ MED 383;3 NEJM.ORG JULY 16, 2020

The New England Journal of Medicine

Downloaded from nejm.org at Ghent University Library on July 24, 2020. For personal use only. No other uses without permission.

Copyright @ 2020 Massachusetts Medical Society. All rights reserved. 


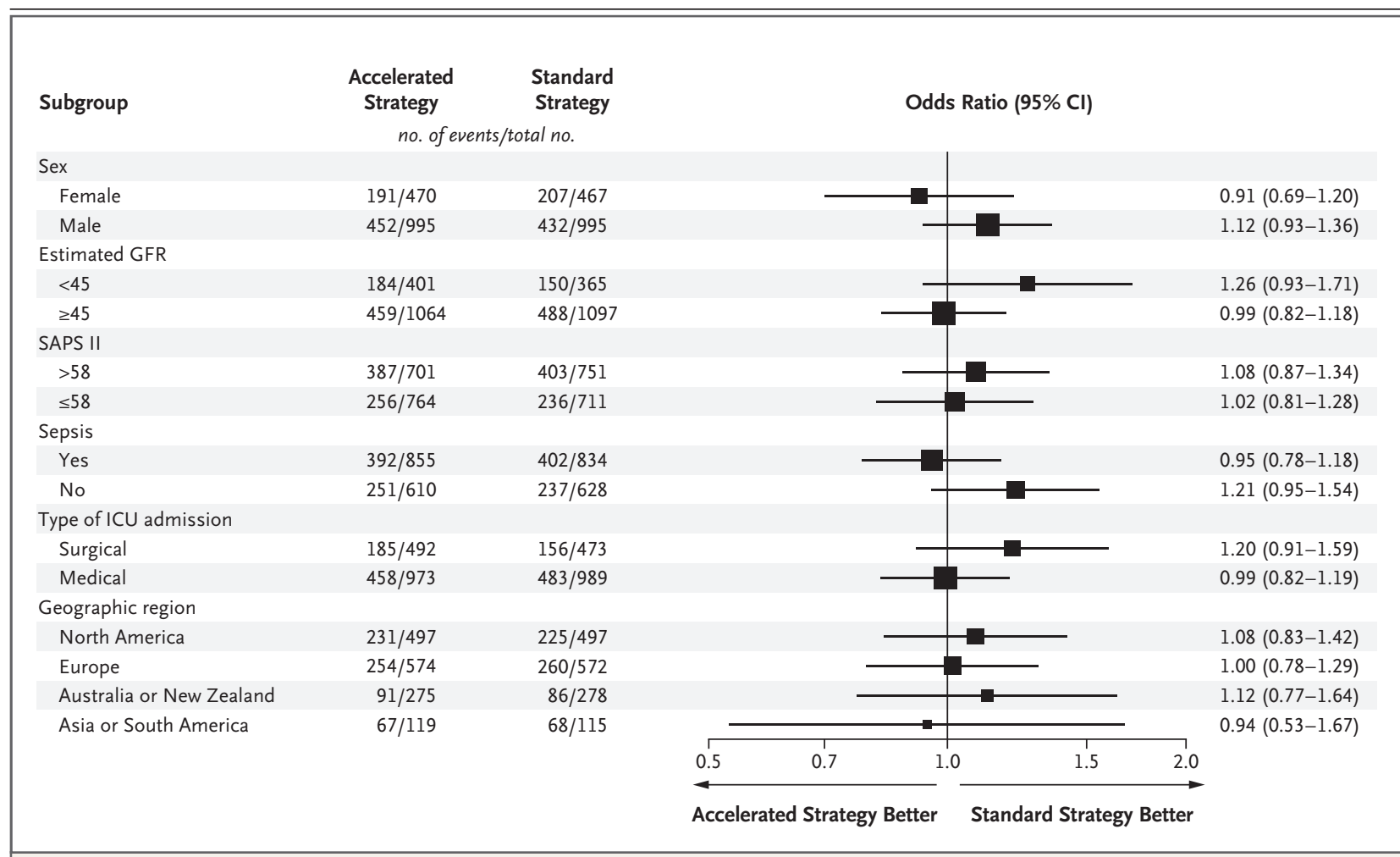

Figure 2. Subgroup Analyses.

Shown is a forest plot of the risk of the primary outcome (death from any cause at 90 days) in the accelerated-strategy group and the standard-strategy group. The size of the square representing the odds ratio reflects the relative number of patients in each subgroup. Results for the Simplified Acute Physiology Score (SAPS) II range from 0 to 163, with higher scores indicating more severe disease and a higher risk of death. GFR denotes glomerular filtration rate, and ICU intensive care unit.

SENSITIVITY ANALYSIS OF THE PRIMARY OUTCOME

In the as-treated analysis, there was no evidence of a between-group difference in 90-day mortality. Death was reported in 623 of 1418 patients (43.9\%) in the accelerated-strategy group and in 659 of $1509(43.7 \%)$ in the standard-strategy group (unadjusted odds ratio, 1.01; 95\% CI, 0.87 to 1.17$)$.

\section{ADVERSE EVENTS}

Adverse events occurred in 346 of 1503 patients (23.0\%) in the accelerated-strategy group and in 245 of 1489 patients $(16.5 \%)$ in the standardstrategy group (risk ratio, 1.40; 95\% CI, 1.21 to 1.62; $\mathrm{P}<0.001$ ) (Table 3). Hypotension and hypophosphatemia were the most common adverse events with a significant between-group difference. There was no difference in serious adverse events between the two strategies.

\section{DISCUSSION}

In this large, multinational, randomized trial, critically ill patients with severe acute kidney injury who received an accelerated strategy for the initiation of renal-replacement therapy did not have a lower risk of death at 90 days than those who received a standard strategy. This finding clarifies a long-standing clinical dilemma regarding the treatment strategy for critically ill patients with acute kidney injury who have no overt complications that would mandate the immediate initiation of renal-replacement therapy. ${ }^{20}$ Observational studies using various biochemical thresholds as surrogates for the timing of initiation and a single-center randomized trial that enrolled primarily surgical patients suggested that earlier renal-replacement therapy resulted in a lower mortality than delayed therapy.,21-23 


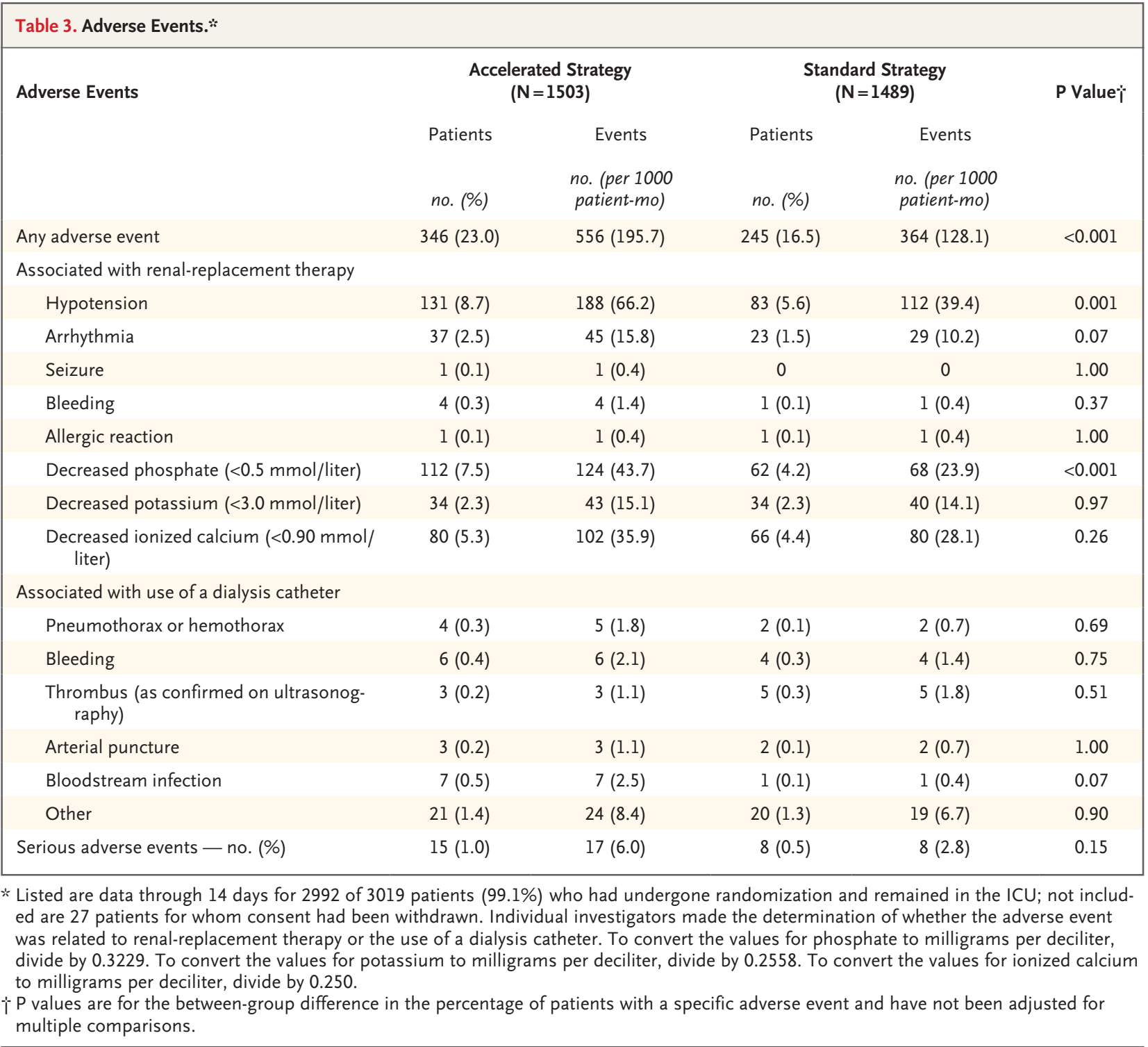

Conversely, two multicenter, randomized trials, including one that exclusively involved patients with septic shock, did not show a lower risk of death with an early strategy than with a delayed strategy for the initiation of renal-replacement therapy. ${ }^{4,5}$ In these two trials, eligibility was tied to fulfilling consensus-defined serum creatinine levels and urine-output thresholds for severe acute kidney injury, with renal-replacement therapy initiated promptly after this determination in the early-therapy group. In the delayed-therapy group, the initiation of renal-replacement therapy was mandated for patients in whom acute kidney injury persisted for 48 to 72 hours or in whom metabolic or fluid complications developed. In the two trials, a sizable percentage of patients in the delayed-therapy group did not receive renal-replacement therapy because they either recovered kidney function or died.

In our trial, we aimed to enrich our patient population by incorporating clinical equipoise to guide eligibility, rather than relying on defined intervals after the patients fulfilled consensus criteria for severe acute kidney injury. This process excluded more than 7000 patients who had been rated by clinicians as being appropriate candidates for emergency renal-replacement therapy or likely to have imminent recovery of kidney 
function. Furthermore, triggers for the initiation of renal-replacement therapy in our standardstrategy group were not directed by thresholds for severity of acute kidney injury or defined time intervals. ${ }^{4,57}$ Our trial also provided evidenceinformed recommendations for the prescription of renal-replacement therapy and adopted a pragmatic approach to reflect current practice. Similar to our pilot work and previous trials, a substantial percentage of the patients in the standard-strategy group did not receive renalreplacement therapy, which highlights the challenge faced by clinicians in predicting worsening acute kidney injury in critically ill patients. ${ }^{4-6}$ Despite these differences in trial design, we did not observe a lower 90-day mortality with an accelerated strategy, a finding that was robust in adjusted and sensitivity analyses.

A greater percentage of survivors who received the accelerated strategy were dependent on renalreplacement therapy at 90 days and had adverse events. This finding suggests that greater exposure to renal-replacement therapy, possibly modified according to baseline risk (e.g., the presence of chronic kidney disease) or mediated by iatrogenic factors (e.g., hypotension), may compromise kidney repair and the return of endogenous kidney function. ${ }^{24-26}$

There are noteworthy strengths of our trial. First, our large sample size afforded the ability to detect a clinically important difference in mortality between accelerated and standard initiation of renal-replacement therapy. Second, the recruitment of patients from a wide spectrum of ICUs in several countries enabled broad generalizability. Third, our trial deliberately enrolled patients for whom the decision on the initiation of renal-replacement therapy was genuinely uncertain by excluding those for whom treating clinicians felt a lack of equipoise. ${ }^{6}$

Our trial also has limitations. First, we recognize that equipoise is relatively subjective and that the perception of equipoise may be modified by individual clinician bias. By allowing clinicians to use their judgment in confirming full eligibility, we may have introduced patient heterogeneity into the trial. However, we did not observe evidence of substantial heterogeneity of treatment effect across subgroups, including illness severity and geographic region. Second, although the protocol provided recommendations on when to start renal-replacement therapy in patients in the standard-strategy group, the broad discretion that was afforded to clinicians may have resulted in variable initiation times. Third, adverse events were more frequent with the accelerated strategy, although this result may be partly attributed to the prespecified focus on reporting of events related to renal-replacement therapy and to the larger number of patient-days of such therapy with this strategy.

In conclusion, in critically ill patients with severe acute kidney injury, an accelerated strategy for the initiation of renal-replacement therapy did not result in a lower mortality at 90 days than a standard strategy.

A data sharing statement provided by the authors is available with the full text of this article at NEJM.org.

Supported by grants from the Canadian Institutes of Health Research (MOP142296 and 389635), the Canadian Institutes of Health Research in partnership with Baxter (IPR-139081), the National Health Medical Research Council of Australia (1127121), the Health Research Council of New Zealand (17/204), and the Health Technology Assessment Program of the United Kingdom National Institutes of Health Research (17/42/74). In 2017, the trial was adopted by the United Kingdom National Institutes for Health Research as a portfolio study. The Canadian Critical Care Trials Group is supported by a grant from the Canadian Institutes of Health Research (138094). Dr. Bagshaw is supported by a Canada Research Chair in Critical Care Nephrology. Dr. Nichol is supported by a Health Research Board of Ireland Clinical Trial Network award. Dr. Schneider is supported by a grant from the Leenaards Foundation. Dr. Young is supported by a Clinical Practitioner Research Fellowship from the Health Research Council of New Zealand.

Disclosure forms provided by the authors are available with the full text of this article at NEJM.org.

We thank Drs. Damon Scales and Robert Fowler for their review of a previous version of the manuscript.

\section{APPENDIX}

The affiliations of the members of the writing committee are as follows: the Department of Critical Care Medicine, Faculty of Medicine and Dentistry, University of Alberta and Alberta Health Services, Edmonton (S.M.B.), the Division of Nephrology (R.W.), St. Michael's Hospital and the University of Toronto, Li Ka Shing Knowledge Institute (R.W., B.R.C., O.M.S., K.E.T.), Department of Medicine (R.W., O.M.S.), and Applied Health Research Centre (B.R.C., K.E.T.), St. Michael's Hospital, the Dalla Lana School of Public Health (K.E.T.), the Institute of Health Policy, Management, and Evaluation (R.W., B.R.C.), University of Toronto, and the Department of Critical Care Medicine, Sunnybrook Health Sciences Centre and the University of Toronto (N.K.J.A.), Toronto, the Department of Medicine, Université de Sherbrooke and Centre de Recherche du Centre Hospitalier Universitaire (CHU) de Sherbrooke, Sherbrooke, QC (F.L.), the Division of Critical Care, Juravinski Hospital, McMaster University, Hamilton, ON (B.R.), and the Division of Nephrology, London Health Sciences Centre, London, ON (M.W.) - all in Canada; the Department of Intensive Care, Austin Hospital and Royal Melbourne Hos- 
pital, School of Medicine, University of Melbourne, Australian and New Zealand Intensive Care Research Centre, School of Public Health and Preventive Medicine, Monash University (R.B., A.D.N.), Melbourne, VIC, and the George Institute for Global Health, Concord Clinical School, Faculty of Medicine, University of Sydney, Sydney (M.P.G., A.Y.W.) - both in Australia; the Institute of Primary Health Care, University of Bern, Bern (B.R.C.), and the Department of Critical Care Medicine, CHU Vaudois, Lausanne (A.G.S.) - both in Switzerland; Hôpital Louis Mourier (D.D.) and Université Léonard de Vinci (S.G.), INSERM Unité UMR S1155, Sorbonne Université and Université de Paris, Paris, Hôpital Avicenne, Bobigny (S.G.), and Hôpital Universitaire François Mitterrand, Lipness Team, INSERM Research Center Lipids, Nutrition, Cancer-Unité Mixte de Recherche 1231 and Laboratoire d'Excellence LipSTIC, Centre d'Investigation Clinique-Epidemiologie Clinique, CHU Dijon-Bourgogne, and INSERM Centre d'Investigation Clinique 1432, Université de Bourgogne, Dijon (J.-P.Q.) - all in France; the Department of Critical Care Medicine, Peking Union Medical College Hospital, Beijing (B.D.), and the Department of Critical Care Medicine, Zhongda Hospital Southeast University, Nanjing (H.Q.) — both in China; the Department of Intensive Care, University of Ghent, Ghent, Belgium (E.A.H.); the Division of Intensive Care and Emergency Medicine, Department of Internal Medicine, Medical University Innsbruck, Innsbruck, Austria (M.J.); Vita Salute San Raffaele University and IRCCS San Raffaele Scientific Institute, Milan (G.L.); the Divisions of Nephrology and Critical Care Medicine, University of California, San Francisco, San Francisco (K.D.L.); the Wellcome-Wolfson Institute for Experimental Medicine, Queen's University, and the Regional Intensive Care Unit, Royal Victoria Hospital, Belfast (D.F.M.), and King's College London, Guy's and St. Thomas' Hospital, London (M.O.) both in the United Kingdom; the Cardiothoracic and Vascular Intensive Care Unit, Auckland City Hospital, Auckland (S.P.M.), and the Medical Research Institute of New Zealand (S.P.M.) and the Intensive Care Unit, Wellington Regional Hospital and Medical Research Institute of New Zealand (P.Y.), Wellington — both in New Zealand; the Division of Nephrology, Bone and Mineral Metabolism, University of Kentucky, Lexington (J.A.N.); University College Dublin Clinical Research Centre at St. Vincent's University Hospital, Dublin (A.D.N.); the Division of Nephrology, University of Pittsburgh, and Veterans Affairs Pittsburgh Healthcare System, Pittsburgh (P.M.P.); the Department of Intensive Care, University of Helsinki, and Helsinki University Hospital, Helsinki (V.P., S.V.); Hospital de Clínicas de Porto Alegre, Porto Alegre, Brazil (F.T.); and the Department of Anesthesiology, Intensive Care and Pain Medicine, University Hospital Münster, Münster, Germany (A.Z.).

\section{REFERENCES}

1. Hoste EA, Bagshaw SM, Bellomo R, et al. Epidemiology of acute kidney injury in critically ill patients: the multinational AKI-EPI study. Intensive Care Med 2015; 41:1411-23.

2. Bagshaw SM, Darmon M, Ostermann $\mathrm{M}$, et al. Current state of the art for renal replacement therapy in critically ill patients with acute kidney injury. Intensive Care Med 2017;43:841-54.

3. Tolwani A. Continuous renal-replace ment therapy for acute kidney injury. N Engl J Med 2012;367:2505-14

4. Barbar SD, Clere-Jehl R, Bourredjem A, et al. Timing of renal-replacement therapy in patients with acute kidney injury and sepsis. N Engl J Med 2018;379:1431-42. 5. Gaudry S, Hajage D, Schortgen F, et al. Initiation strategies for renal-replacement therapy in the intensive care unit. N Eng J Med 2016;375:122-33.

6. Wald R, Adhikari NKJ, Smith OM, et al. Comparison of standard and accelerated initiation of renal replacement therapy in acute kidney injury. Kidney Int 2015;88: 897-904.

7. Zarbock A, Kellum JA, Schmidt C, et al. Effect of early vs delayed initiation of renal replacement therapy on mortality in critically ill patients with acute kidney injury: the ELAIN randomized clinical trial. JAMA 2016;315:2190-9.

8. Wald R, Gallagher M, Bagshaw SM. Shedding new light on an old dilemma: two trials examining the timing of renal replacement therapy initiation in acute kidney injury. Am J Kidney Dis 2017;69:14-7. 9. Fayad AII, Buamscha DG, Ciapponi A Timing of renal replacement therapy initiation for acute kidney injury. Cochrane Database Syst Rev 2018;12:CD010612.
10. Libório AB, Leite TT, Neves FM, Teles F, Bezerra CT. AKI complications in critically ill patients: association with mortality rates and RRT. Clin J Am Soc Nephrol 2015;10:21-8.

11. The STARRT-AKI Investigators. Statistical analysis plan for the Standard versus Accelerated Initiation of Renal Replacement Therapy in Acute Kidney Injury (STARRT-AKI) trial. Crit Care Resusc 2019; 21:162-70.

12. The STARRT-AKI Investigators. STandard versus Accelerated initiation of Renal Replacement Therapy in Acute Kidney Injury: study protocol for a multi-national, multi-center, randomized controlled trial. Can J Kidney Health Dis 2019;6: 2054358119852937.

13. Kidney Disease: Improving Global Outcomes (KDIGO) Acute Kidney Injury Work Group. KDIGO clinical practice guideline for acute kidney injury. Kidney Int Suppl 2012;2:1-138

14. Semler MW, Self WH, Rice TW. Balanced crystalloids versus saline in critically ill adults. N Engl J Med 2018;378: 1951.

15. Schoenfeld DA, Bernard GR. Statistical evaluation of ventilator-free days as an efficacy measure in clinical trials of treatments for acute respiratory distress syndrome. Crit Care Med 2002;30:1772-7. 16. Herdman M, Gudex C, Lloyd A, et al. Development and preliminary testing of the new five-level version of EQ-5D (EQ-5D-5L). Qual Life Res 2011;20:172736.

17. Nisula S, Kaukonen K-M, Vaara ST, et al. Incidence, risk factors and 90-day mortality of patients with acute kidney injury in Finnish intensive care units: the
FINNAKI study. Intensive Care Med 2013; 39:420-8.

18. Singer M, Deutschman CS, Seymour $\mathrm{CW}$, et al. The Third International Consensus Definitions for Sepsis and Septic Shock (Sepsis-3). JAMA 2016;315:801-10. 19. The R Project for Statistical Computing. R software package 2008 (www.R -project.org).

20. Parsons FM, Hobson SM, Blagg CR, McCracken BH. Optimum time for dialysis in acute reversible renal failure: description and value of an improved dialyser with large surface area. Lancet 1961;1:129-34. 21. Bagshaw SM, Uchino S, Bellomo R, et al. Timing of renal replacement therapy and clinical outcomes in critically ill patients with severe acute kidney injury. J Crit Care 2009;24:129-40.

22. Liu KD, Himmelfarb J, Paganini E, et al. Timing of initiation of dialysis in critically ill patients with acute kidney injury. Clin J Am Soc Nephrol 2006;1:915-9.

23. Vaara ST, Reinikainen M, Wald R, Bagshaw SM, Pettilä V, FINNAKI Study Group. Timing of RRT based on the presence of conventional indications. Clin J Am Soc Nephrol 2014;9:1577-85.

24. Yang L, Besschetnova TY, Brooks CR, Shah JV, Bonventre JV. Epithelial cell cycle arrest in G2/M mediates kidney fibrosis after injury. Nat Med 2010;16:535-43.

25. Marants R, Qirjazi E, Grant CJ, Lee TY, McIntyre CW. Renal perfusion during hemodialysis: intradialytic blood flow decline and effects of dialysate cooling. J Am Soc Nephrol 2019;30:1086-95.

26. Conger JD. Does hemodialysis delay recovery from acute renal failure? Semin Dial 1990;3:146-8.

Copyright (C) 2020 Massachusetts Medical Society. 